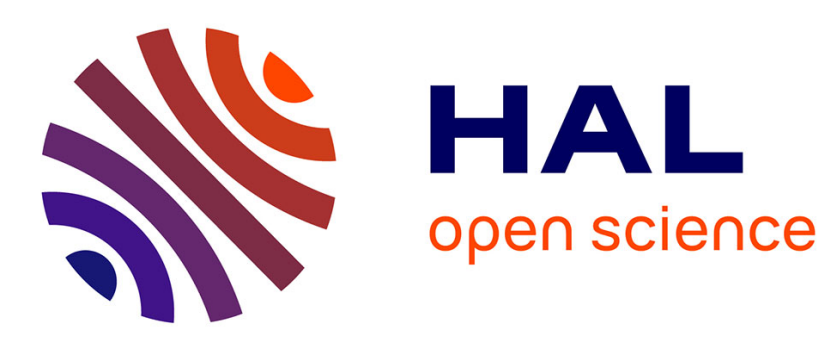

\title{
Calibration of Industrial Robots with Pneumatic Gravity Compensators
}

\author{
Alexandr Klimchik, Anatol Pashkevich, Stéphane Caro, Benoît Furet
}

\section{To cite this version:}

Alexandr Klimchik, Anatol Pashkevich, Stéphane Caro, Benoît Furet. Calibration of Industrial Robots with Pneumatic Gravity Compensators. 2017 IEEE International Conference on Advanced Intelligent Mechatronics (AIM), Jul 2017, Munich, Germany. 10.1109/AIM.2017.8014031 . hal-01757907

\section{HAL Id: hal-01757907 https://hal.science/hal-01757907}

Submitted on 4 Apr 2018

HAL is a multi-disciplinary open access archive for the deposit and dissemination of scientific research documents, whether they are published or not. The documents may come from teaching and research institutions in France or abroad, or from public or private research centers.
L'archive ouverte pluridisciplinaire HAL, est destinée au dépôt et à la diffusion de documents scientifiques de niveau recherche, publiés ou non, émanant des établissements d'enseignement et de recherche français ou étrangers, des laboratoires publics ou privés. 


\title{
Calibration of industrial robots with pneumatic gravity compensators
}

\author{
A. Klimchik, IEEE Member, A. Pashkevich, S. Caro and B. Furet
}

\begin{abstract}
The paper is devoted to the stiffness modeling of heavy industrial robots with pneumatic gravity compensators. The main attention is paid to the identification of elastostatic parameters and calibration accuracy. To reduce impact of the measurement errors, the set of manipulator configurations for calibration experiments is optimized with respect to the proposed performance measure related to the end-effector position accuracy. Advantages of the developed technique are illustrated by dedicated experimental study.
\end{abstract}

Keywords - Industrial robot, stiffness modeling, elastostatic calibration, pneumatic gravity compensator, design of calibration experiments.

\section{INTRODUCTION}

Advancements in shipbuilding and aeronautic industries demand high-precision and high-speed machining of huge hulls and fuselage components. For these tasks, industrial robots are more attractive comparing to conventional CNCmachines because of large and easily extendable workspace, capability to process complex-shape parts and high-speed motion capability. However, processing of modern and contemporary materials, which are widely used in these industries, requires high processing forces affecting robot positioning accuracy [1-3]. To reduce the external force impact on the positioning accuracy, robotic experts usually apply technique that is based on the compliance error estimation via the manipulator stiffness modelling [4-7] and relevant error compensation in the online or offline mode [8-11]. This approach is very efficient if the stiffness and geometric model parameters of the manipulator as well as the external forces are known. To estimate them, additional experimental studies are usually carried out [4, 12-14], which allow user to obtain an extended geometric model suitable for compliance error compensation.

Another practical solution is based on enhancement the robot stiffness by means of increasing the link cross-sections. However, it leads to increasing of the robot components masses causing additional end-effector deflections, which are usually reduced by means of different types of gravity com-

\footnotetext{
*The work presented in this paper was partially funded by Innopolis University and the project Partenariat Hubert Curien SIAM 2016 FranceThailand

A. Klimchik is with Innopolis University, Universitet-skaya St, 1, Innopolis, 420500, Russia (e-mail: a.klimchik@innopolis.ru).

A. Pashkevich is with IMT Atlantique, 4 rue Alfred-Kastler, Nantes 44307, France and with Institut LS2N (e-mail: anatol.pashkevich@imtatlantique.fr).

S. Caro is with Centre National de la Recherche Scientifique (CNRS), France (email: stephane.caro@1s2n.fr)

B. Furet are with University of Nantes, France (e-mails: alexandre.ambieh1@univ-nantes.fr, benoit.furet@univ-nantes.fr).
}

pensators. However, integration of mechanical compensators into manipulator kinematics essentially complicates the stiffness modelling, because conventional serial architecture is transformed into the quasi-serial one that contains a kinematic closed loop.

The stiffness modelling of the industrial manipulators with mechanical gravity compensators is quite a new problem. There are rather limited number of works dealing with the impact of gravity compensators on the manipulator forcedeflection relation $[13,15,16]$, while there are some work devoted to the compensator design $[17,18]$ and softwarebased balancing solutions $[19,20]$. In contrast, for conventional strictly serial manipulators $[6,21,22]$ and strictly parallel mechanisms [7, 23-27] there were developed a number of methods for the stiffness analysis. At the same time, only limited number of works deals with stiffness modelling of socalled quasi-serial architectures incorporating internal closed-loops [15, 28-31]. To our knowledge, the simplest and efficient way to take into account the influence of gravity compensator is utilization of non-linear virtual springs in the frame of the conventional VJM technique $[7,32,33]$. This approach was originally proposed in our previous works $[15$, $16]$ and successfully applied to the manipulators the springbased gravity compensators. However, some additional efforts are required to adapt it to the case of robots with pneumatic compensators, which progressively replace their counterparts in new models of heavy industrial robots available on the market.

This paper proposes a new modification of the VJMbased stiffness modelling technique for the quasi-serial industrial manipulators with a pneumatic gravity compensator that creates a kinematic closed-loop violating the stiffness modelling principles, which are used for pure serial robot architecture. The main attention is paid to the identification of the model parameters and calibration experiment planning. The developed approach is confirmed by the experimental results that deal with compliance error compensation for robotic cell employed in manufacturing of large dimensional components.

To address these problems the remainder of the paper is organized as follows. Section 2 presents the stiffness modelling for pneumatic gravity compensator. Section 3 is devoted to the elastostatic calibration and design of calibration experiments. Section 4 deals with experimental study. Section 5 summarizes the main contributions of the paper. 


\section{MECHANICS OF PNEUMATIC GRAVITY COMPENSATOR}

The mechanical structure and principal components of pneumatic gravity compensator considered here is presented in Fig. 1a; its equivalent model is shown in Fig. 1b. The mechanical compensator is a passive mechanism incorporating a constant cross-section cylinder and a constant volume gas reservoir. The volume occupied by the gas linearly depends on the piston position that defines the internal pressure of the cylinder. It is clear that this mechanism can be treated as a non-linear virtual spring influencing on the manipulator stiffness behavior. It is worth mentioning that in general case the gas temperature has impact on the pressure inside the tank, which defines the compensating force. Nevertheless, one can assume that in the case of continuous or periodical manipulator movements the gas temperature remains almost constant, i.e. the process of the gas compression-decompression can be assumed to be the isothermal one.

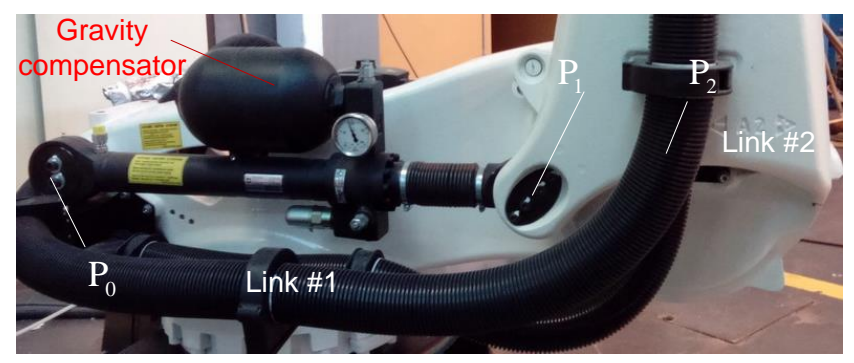

(a) Pneumatic gravity compensator of robot KUKA KR-210 TM

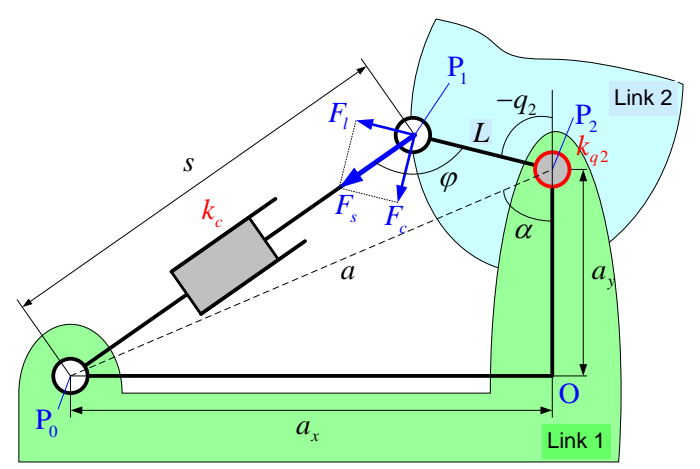

(b) model of gravity compensator

Figure 1. Pneumatic gravity compensator and its mode

In the frame of the manipulator model, the compensator is attached to the first and second links that creates a closedloop acting on the second actuated joint. This particularity allows us to adapt the conventional stiffness model of the serial manipulator (with constant joint stiffness matrix $\mathbf{K}_{\theta}$ ) by introducing the configuration dependent joint stiffness matrix $\mathbf{K}_{\theta}(\mathbf{q})$ that takes into account the compensator impact and depends on the vector of actuated coordinates $\mathbf{q}$. In this case, the Cartesian stiffness matrix $\mathbf{K}_{\mathrm{C}}$ of the robotic manipulator [22] can be presented in the following form

$$
\mathbf{K}_{\mathrm{C}}=\left(\mathbf{J}_{\theta} \cdot \mathbf{K}_{\theta}^{-1}(\mathbf{q}) \cdot \mathbf{J}_{\theta}^{\mathrm{T}}\right)^{-1}
$$

where $\mathbf{J}_{\theta}$ is the Jacobian with respect to the virtual joint coordinates $\boldsymbol{\theta}$ (in the case of industrial robots it is usually equivalent to the kinematic Jacobian computed with respect to actuated coordinates $\mathbf{q}$ ). Thus, to obtain the stiffness model of the industrial robot with the pneumatic gravity compensator it is required to determine the non-linear joint stiffness matrix $\mathbf{K}_{\theta}(\mathbf{q})$ describing elasticity of both actuators and the gravity compensation mechanism. It should be mentioned that in the majority of works devoted to the stiffness analysis of the serial manipulators the matrix $\mathbf{K}_{\theta}$ is assumed to be a constant and strictly diagonal one [22, 26, 34].

To find the desired matrix $\mathbf{K}_{\theta}(\mathbf{q})$, let us consider the compensator geometry in detail. As follows from Fig 1b, the compensator geometrical model contains three principal node points $\mathrm{P}_{0}, \mathrm{P}_{1}, \mathrm{P}_{2}$., where $\mathrm{P}_{0}, \mathrm{P}_{1}$ define the passive joint rotation axes and $\mathrm{P}_{2}$ defines the second actuated joint axis. In this model, two distances $\left|P_{1}, P_{2}\right|,\left|P_{0}, P_{2}\right|$ are constants, while the third one $\left|P_{0}, P_{1}\right|$ varies with the robot motions and non-linearly depends on the angle $q_{2}$. Below, this distances are denoted as follows: $L=\left|P_{1}, P_{2}\right|, a=\left|P_{1}, P_{2}\right|, \quad s=\left|P_{0}, P_{1}\right|$. In addition, let us introduce parameters $\alpha, \varphi, a_{x}$ and $a_{y}$ defining relevant locations of points $\mathrm{P}_{0}, \mathrm{P}_{1}, \mathrm{P}_{2}$ (see Fig. 1b). This allows us to compute the compensator length $s$ using the following expression

$$
s^{2}=a^{2}+L^{2}+2 \cdot a \cdot L \cdot \cos \left(\alpha-q_{2}\right)
$$

which defines the non-linear function $s\left(q_{2}\right)$.

For this geometry, the impact of the gravity compensator can be taken into account by replacing the considered quasiserial architecture by the serial one, where the second joint stiffness coefficient is modified in order to include elasticity of both the actuator and compensator. To find relevant nonlinear expression for this coefficient, let us present the static torque in the second joint $M_{2}$ as a geometric sum of two components. The first of them is caused by the deflection $\delta q_{2}$ in the mechanical transmission of the second actuated joint and can be expressed in a usual way as $M_{q_{2}}=K_{q_{2}} \delta q_{2}$, where $K_{q_{2}}$ is the stiffness coefficient. The second component can be presented as $M_{C}=F_{S} \cdot L \cdot \sin \varphi$, where $F_{S}$ is the force generated by the gravity compensator. It is clear that $\sin \varphi$ can be computed from the triangle $\Delta P_{0} P_{1} P_{2}$ using the sines theorem: $\sin \varphi=(a / s) \cdot \sin \left(\alpha-q_{2}\right)$. The latter allows us to express the torque $M_{2}$ in the following form:

$$
M_{2}=K_{q_{2}} \delta q_{2}+F_{S} \cdot L \cdot \frac{a}{s} \sin \left(\alpha-q_{2}\right)
$$

where both the force $F_{S}$ and the compensator length $s$ depend on the joint variable $q_{2}=q_{2}^{0}+\delta q_{2}$.

To find the compensating force $F_{S}$, let us use the isothermal process assumption that yields the relation $P \cdot V=$ const, where $P$ is the tank pressure, $V=A \cdot\left(s-s_{0}\right)+V_{0}$ is the corresponding internal volume, $A$ is the piston area, $s_{0}$ is compensator link length corresponding to zero compensating force and $V_{0}$ is the tank volume corresponding to the atmospheric pressure $P_{0}=1.01 \cdot 10^{5} \mathrm{~Pa}$. These assumptions allow us to express the tank pressure as

$$
P=\frac{P_{0} \cdot V_{0}}{A \cdot\left(s-s_{0}\right)+V_{0}}
$$

Taking into account that compensating force $F_{S}$ depends on the internal and external pressure difference and is computed as $F_{S}=\left(P-P_{0}\right) \cdot A$, one can rewrite expression (3) as 


$$
M_{2}=K_{q_{2}} \delta q_{2}-P_{0} \frac{A \cdot\left(s-s_{0}\right)}{A \cdot\left(s-s_{0}\right)+V_{0}} \cdot L \cdot \frac{a}{s} \sin \left(\alpha-q_{2}\right)
$$

and present in a more compact form

$$
M_{2}=K_{q_{2}} \delta q_{2}-P_{0} \cdot L \cdot a \cdot \frac{\sin \left(\alpha-q_{2}\right)}{s} \frac{s-s_{0}}{s-s_{V}}
$$

where a constant $s_{V}=V_{0} / A-s_{0}$ is the equivalent distance.

Further, after computing the partial derivative $\partial M_{2} / \partial q_{2}$ and using eq. (2) for $\partial s / \partial q_{2}=a \cdot L / s \cdot \sin \left(\alpha-q_{2}\right)$, the desired aggregated stiffness coefficient is presented in the form

$$
\begin{aligned}
K_{2}\left(q_{2}\right) & =K_{q_{2}}-\frac{P_{0} \cdot L \cdot a}{s^{2}\left(s-s_{V}\right)}\left(a \cdot L \cdot \sin ^{2}\left(\alpha-q_{2}\right) \cdot \frac{s_{0}-s_{V}}{s-s_{V}}+\right. \\
& \left.+\left(-s^{2} \cdot \cos \left(\alpha-q_{2}\right)-a \cdot L \cdot \sin ^{2}\left(\alpha-q_{2}\right)\right) \cdot \frac{s-s_{0}}{s}\right)
\end{aligned}
$$

which is obviously highly non-linear with respect to manipulator configuration (here, $s$ is also a non-linear function of $q_{2}$ ). Nevertheless, it allows us to compute an relevant stiffness coefficient $K_{2}$ for the equivalent serial chain and directly apply eq. (1) to evaluate stiffness of the quasi-serial manipulator with pneumatic gravity compensator.

It should be mentioned that in practice the compensator parameters $s_{0}, s_{V}$ and actuator stiffness coefficients $K_{1}, K_{2}, K_{3}, \ldots, K_{6}$ are usually not given in the robot datasheets, so they should be identified via dedicated experimental study. For this reason, the following Section focuses on the identification of this extended set of the manipulator elastostatic parameters.

\section{ELASTOSTATIC PARAMETERS IDENTIFICATION}

\section{A. Methodology}

In the frame of the VJM-based modelling approach developed for serial kinematic chains [22, 32] and adapted here for the case of quasi-serial manipulators with pneumatic gravity compensators, the desired stiffness model parameters describe elasticity of the virtual springs located in the actuated joints of the manipulator, and also compensator parameters $s_{0}, s_{V}$ of defining preloading of the compensator spring and the equivalent distance for the tank volume $V_{0}$. In the frame of this model, let us denote the manipulator joint compliances as $k_{\theta}, j=\overline{1,6}$ (which are inverse values to the joint stiffness coefficients $K_{1}, K_{2}, K_{3}, \ldots, K_{6}$ used in previous section) and the compensator elastic parameters as $s_{0}, s_{V}$.

To find the desired set of elastic parameters, the robotic manipulator sequentially passes through several measurement configurations where the external loading is applied to the specially designed end-effector presented in Fig. 2 (it allows us to generate both forces and torques applied to the manipulator). Using the absolute measurement system (the laser tracker Leica AT900, the Cartesian coordinates of the reference points are measured twice, before and after loading. To increase identification accuracy, it is reasonable to have several markers on the end effector (reference points) and to apply the loading of the maximum allowed magnitude. It should be mentioned that to avoid singularities caused by numerical routines, the external force/torque directions should not be the same for all calibration experiments (while from the practical view point the mass-based gravity loading is the most attractive). Thus, the calibration experiments yield the dataset that includes values of the manipulator joint coordinates $\left\{\mathbf{q}_{i}\right\}$, applied forces/torques $\left\{\mathbf{F}_{i}\right\}$ and corresponding deflections of the reference points $\left\{\Delta \mathbf{p}_{i}\right\}$. Using these data, it is required to identify the manipulator elastostatic parameters of $k_{\theta_{j}}, j=\overline{1,6}$ and gravity compensator parameters $s_{0}, s_{V}$.

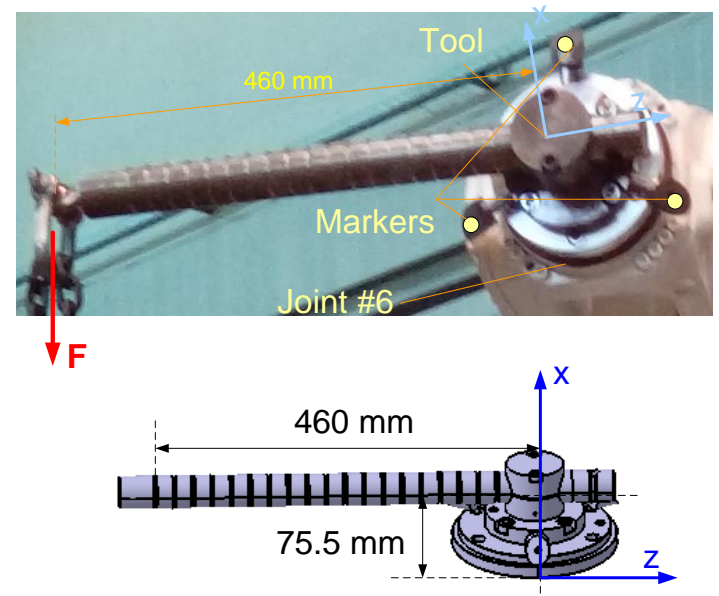

Figure 2. End-effector used for the elastostatic calibration experiments and it model

\section{B. Identification algorithm}

To take into account the compensator influence while using classical approach developed for strictly serial manipulators without compensators [6], it was proposed below to use in the second joint an equivalent virtual spring with nonlinear stiffness, which depends on the joint coordinate $q_{2}$ (see eq. (1)). Using this idea, it is convenient to consider several aggregated compliances $k_{\theta}$ corresponding to each different value of angle $q_{2}$. This idea allows us to linearize the identification equations with respect to extended set of model parameters and that can be easily solved using standard least-square technique.

Let us denote this extended set of desired parameters as $k_{1},\left(k_{21}, k_{22} \ldots\right), k_{3}, \ldots, k_{6}$ and collect them in the vector $\mathbf{k}$. In this case the linearize force-deflection relation with respect to this vector can be present in the following form

$$
\Delta \mathbf{p}_{i}=\mathbf{B}_{i}^{(p)} \mathbf{k}
$$

where $\Delta \mathbf{p}_{i}$ is the vector of the end-effector displacements under the external loading $\mathbf{F}_{i}$, matrices $\mathbf{B}_{i}^{(p)}$ are composed of the elements of the matrix

$$
\mathbf{A}_{i}=\left[\mathbf{J}_{1 i} \mathbf{J}_{1 i}{ }^{T} \mathbf{F}_{i}, \ldots, \mathbf{J}_{n i} \mathbf{J}_{n i}{ }^{T} \mathbf{F}_{i}\right] \quad(i=\overline{1, m})
$$

that is usually used in stiffness analysis of serial manipulators. Here, $\mathbf{J}_{n i}$ denotes the manipulator Jacobian column, superscript '(p)' stands for the Cartesian coordinates (position without orientation). Transformation from $\mathbf{A}_{i}$ to $\mathbf{B}_{i}^{(p)}$ is ra- 
ther trivial and is based on the extraction from $\mathbf{A}_{i}$ the first three lines and inserting in it several zero columns.

In this case, the elastostatic parameters identification can be reduced to the following optimization problem

$$
F=\sum_{i=1}^{m}\left(\mathbf{B}_{i}^{(p)} \mathbf{k}-\Delta \mathbf{p}_{i}\right)^{T}\left(\mathbf{B}_{i}^{(p)} \mathbf{k}-\Delta \mathbf{p}_{i}\right) \rightarrow \min _{k_{j}, k_{c}, \rho_{0}}
$$

which yields to the following solution

$$
\mathbf{k}=\left(\sum_{i=1}^{m} \mathbf{B}_{i}^{(p)^{T}} \mathbf{B}_{i}^{(p)}\right)^{-1} \cdot\left(\sum_{i=1}^{m} \mathbf{B}_{i}^{(p)^{T}} \Delta \mathbf{p}_{i}\right)
$$

where the parameters $k_{1}, k_{3}, \ldots, k_{6}$ describe the compliance of the virtual joints $\# 1, \# 3, \ldots \# 6$, while the rest of them $k_{21}, k_{22} \ldots$ present an auxiliary dataset allowing to separate the compliance of the joint \#2 and the compensator parameters $s_{0}, s_{V}$. Using eq. (7), the desired optimization problem can be written as

$$
\begin{aligned}
& \sum_{i=1}^{m_{q}}\left[K_{q_{2}}-\frac{P_{0} \cdot L \cdot a}{s_{i}^{2}\left(s_{i}-s_{V}\right)}\left(a \cdot L \cdot \sin ^{2}\left(\alpha-q_{2 i}\right) \cdot \frac{s_{0}-s_{V}}{s_{i}-s_{V}}+\right.\right. \\
& \left.\left.+\left(-s_{i}^{2} \cos \left(\alpha-q_{2}\right)-a L \sin ^{2}\left(\alpha-q_{2 i}\right)\right) \cdot \frac{s_{i}-s_{0}}{s}\right)\right] \rightarrow \min _{K_{q_{2}}, s_{0}, s_{V}}
\end{aligned}
$$

where $m_{q}$ is the number of different angles $q_{2}$ in the experimental data. It is obvious that eq. (12) is highly non-linear and can be solved numerically only.

Thus, the proposed modification of the previously developed calibration technique allows us to find the manipulator and compensator parameters. An open question, however, is how to find the set of measurement configurations that ensure the lowest impact of the measurement noise.

\section{Design of calibration experiments}

The goal of the calibration experiment design is to select a set of robot configurations/external loadings $\left\{\mathbf{q}_{i}, \mathbf{F}_{i}\right\}$ that ensure the best identification accuracy. The key issue is to rang plans of experiments in accordance with selected performance measure. This problem is well known in the classical regression analysis; however, the results are not suitable for non-linear case of the elastostatic calibration and require additional efforts. Here, an industry oriented performance measure is used, which evaluates the calibration plan quality $[13,15]$. Its physical meaning is the robot positioning accuracy (under the loading), which is achieved after compliance error compensation based on the identified elastostatic parameters.

Assuming that experiments include measurement errors $\boldsymbol{\varepsilon}_{i}$, covariance matrix for the parameters $\mathbf{k}$ can be expressed as

$$
\begin{aligned}
\operatorname{cov}(\mathbf{k}) & =\left(\sum_{i=1}^{m} \mathbf{B}_{i}^{(p)^{T}} \mathbf{B}_{i}^{(p)}\right)^{-1} \\
\times & \mathrm{E}\left(\sum_{i=1}^{m} \mathbf{B}_{i}^{(p)^{T}} \boldsymbol{\varepsilon}_{i}^{T} \boldsymbol{\varepsilon}_{i} \mathbf{B}_{i}^{(p)}\right)\left(\sum_{i=1}^{m} \mathbf{B}_{i}^{(p)^{T}} \mathbf{B}_{i}^{(p)}\right)^{-1}
\end{aligned}
$$

Following independent identically distributed assumption with zero expectation and standard deviation $\sigma^{2}$ for the measurement errors, expression (13) can be simplified to

$$
\operatorname{cov}(\mathbf{k})=\sigma^{2}\left(\sum_{i=1}^{m} \mathbf{B}_{i}^{(p)^{T}} \mathbf{B}_{i}^{(p)}\right)^{-1}
$$

Hence, the impact of the measurement errors on the accuracy of the identified parameters $\mathbf{k}$ is defined by the matrix $\sum_{i=1}^{m} \mathbf{B}_{i}^{(p)^{T}} \mathbf{B}_{i}^{(p)}$ (in regression analysis it is known as the information matrix).

It is evident that in industrial practice the most important issue is not the parameters identification accuracy, but their impact on the robot positioning accuracy. Considering that the end-effector accuracy varies throughout the workspace and highly depends on the manipulator configuration, it is proposed to evaluate the calibration accuracy in a typical manipulator configuration ("test-pose") provided by the user. For the most of applications of heavy industrial robots, the test pose is usually related to the typical machining configuration $\mathbf{q}_{0}$ and corresponding external loading $\mathbf{F}_{0}$ related to the corresponding technological process. For the so-called test-pose the mean square value of the positioning error will be denoted as $\rho_{0}^{2}$ and the matrix $\mathbf{A}_{i}^{(p)}$ corresponding to it as $\mathbf{A}_{0}^{(p)}$.

It should be noted that that the proposed approach operates with a specific structure of the parameters included in the vector $\mathbf{k}$, where the second joint is presented by several components $k_{21}, k_{22} \ldots$ while the other joints are described by a single parameter $k_{1}, k_{3} \ldots k_{6}$. This motivates further rearrangement of the vector $\mathbf{k}$ and replacing it by several vectors $\mathbf{k}_{j}=\left(k_{1}, k_{2 j}, k_{3}, \ldots k_{6}\right)$ of size $6 \times 1$. Using this notation, the above mentioned performance measure can be expressed as

$$
\rho_{0}^{2}=\sum_{j=1}^{m_{q}} \mathrm{E}\left(\delta \mathbf{k}_{j}{ }^{T} \mathbf{A}_{0}^{(p)^{T}} \mathbf{A}_{0}^{(p)} \delta \mathbf{k}_{j}\right)
$$

where $\delta \mathbf{k}_{j}$ is the elastostatic parameters estimation error caused by the measurement noise for $q_{2 j}$. Further, after substituting $\delta \mathbf{p}^{T} \delta \mathbf{p}=\operatorname{trace}\left(\delta \mathbf{p} \delta \mathbf{p}^{T}\right)$ and taking into account that $\mathrm{E}\left(\delta \mathbf{k}_{j} \delta \mathbf{k}_{j}{ }^{T}\right)=\operatorname{cov}\left(\mathbf{k}_{j}\right)$, the performance measure $\rho_{0}^{2}$ can be presented as

$$
\rho_{0}^{2}=\sigma^{2} \operatorname{trace}\left(\mathbf{A}_{0}^{(p)} \sum_{j=1}^{m_{q}}\left(\sum_{i=1}^{m} \mathbf{A}_{i}^{j(p)^{T}} \mathbf{A}_{i}^{j(p)}\right)^{-1} \mathbf{A}_{0}^{(p)^{T}}\right)
$$

Based on this performance measure, the calibration experiment design can be reduced to the following optimization problem

$$
\operatorname{trace}\left(\mathbf{A}_{0}^{(p)} \sum_{j=1}^{m_{q}}\left(\sum_{i=1}^{m} \mathbf{A}_{i}^{j(p)^{T}} \mathbf{A}_{i}^{j(p)}\right)^{-1} \mathbf{A}_{0}^{(p)^{T}}\right) \rightarrow \min _{\left\{\mathbf{q}_{i}, \mathbf{F}_{i}\right\}}
$$

subject to $\left\|\mathbf{F}_{i}\right\|<F_{\max }, \quad i=1 . . m$ whose solution gives a set of the desired manipulator configurations and corresponding external loadings. It is evident that its analytical solution can hardly be obtained and a numerical approach is the only reasonable one.

\section{EXPERIMENTAL STUDY}

The developed technique was applied to the elastostatic calibration of robot Kuka KR-120. The parameters to be identified were the compliances $k_{j}$ of the actuated joints and the gravity compensator parameters $s_{0}, s_{V}$. To generate de- 
flections in the actuated joints, the gravity forces $120 \mathrm{~kg}$ were applied to the robot end-effector (see Fig 3). The Cartesian coordinates of three markers located on the tool (see Fig, 2) have been measured before and after the loading.

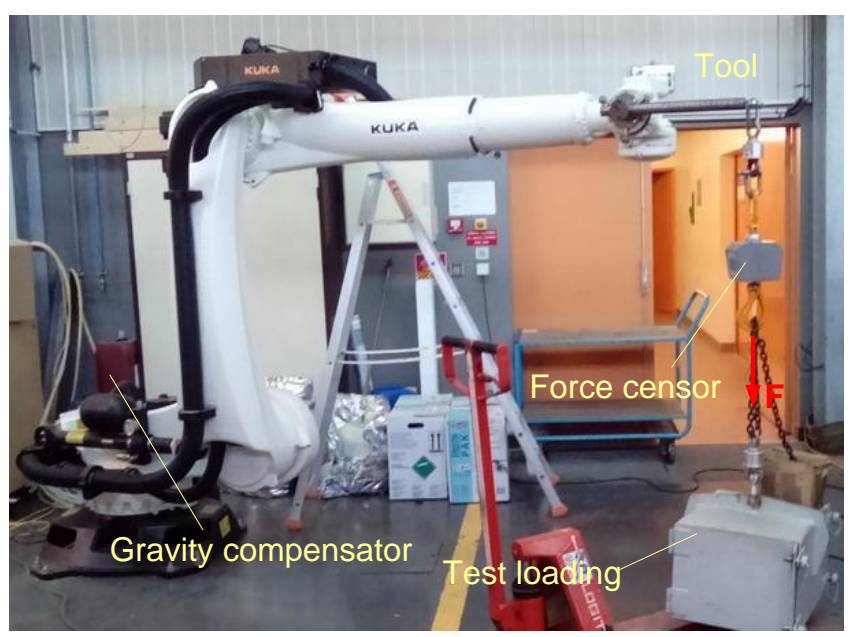

Figure 3. Experimental setup for the identification of the elastostatic parameters.

To find optimal measurement configurations for calibration, the design of experiments was used for six different angles $q_{2}$ that are distributed between the joint limits. For each $q_{2}$ from three to seven optimal measurement configurations were found, which satisfy joint limits and physical constraints related to the possibility carry out experiments. In total 31 different measurement configurations and 186 measurements were considered for the identification, from which 7 physical parameters were obtained. The obtained experimental data have been processed using the identification algorithm presented in Section 3. Identified values for the extended set of joint compliances (for 6 different angles $q_{2}$ ) and their confidence intervals are presented in Table 1. As follows from this results, wrist compliances were identified with lower accuracy. The reason for it is smaller shoulder from the applied external forces comparing with manipulator joints. Relatedly small accuracy of first joint due to a smaller number of measurements in the experiments in which the deflections were generated in the first joint. Further, obtained compliances $\mathrm{k}_{21} \ldots \mathrm{k}_{26}$ were used to estimate pneumonic compensator parameters by solving optimization problem (12). The identified joint compliances can be used to predict robot deformations under the external loading.

TABLE I. ELASTO-STATIC PARAMETERS OF ROBOT KUKA KR-120

\begin{tabular}{|l|l|l|}
\hline \multicolumn{1}{|c|}{ Parameter } & \multicolumn{1}{c|}{ value } & \multicolumn{1}{c|}{ CI } \\
\hline $\mathrm{k}_{1},[\mathrm{rad} \times \mu \mathrm{m} / \mathrm{N}]$ & 1.13 & $\pm 0.15(13.3 \%)$ \\
\hline $\mathrm{k}_{21},[\mathrm{rad} \times \mu \mathrm{m} / \mathrm{N}]$ & 0.34 & $\pm 0.004(1.1 \%)$ \\
\hline $\mathrm{k}_{22},[\mathrm{rad} \times \mu \mathrm{m} / \mathrm{N}]$ & 0.36 & $\pm 0.005(1.4 \%)$ \\
\hline $\mathrm{k}_{23},[\mathrm{rad} \times \mu \mathrm{m} / \mathrm{N}]$ & 0.35 & $\pm 0.005(1.4 \%)$ \\
\hline $\mathrm{k}_{24},[\mathrm{rad} \times \mu \mathrm{m} / \mathrm{N}]$ & 0.28 & $\pm 0.007(2.6 \%)$ \\
\hline $\mathrm{k}_{25},[\mathrm{rad} \times \mu \mathrm{m} / \mathrm{N}]$ & 0.32 & $\pm 0.011(3.6 \%)$ \\
\hline $\mathrm{k}_{26},[\mathrm{rad} \times \mu \mathrm{m} / \mathrm{N}]$ & 0.26 & $\pm 0.007(2.8 \%)$ \\
\hline $\mathrm{k}_{3},[\mathrm{rad} \times \mu \mathrm{m} / \mathrm{N}]$ & 0.43 & $\pm 0.007(1.8 \%)$ \\
\hline $\mathrm{k}_{4},[\mathrm{rad} \times \mu \mathrm{m} / \mathrm{N}]$ & 0.95 & $\pm 0.31(31.8 \%)$ \\
\hline $\mathrm{k}_{5},[\mathrm{rad} \times \mu \mathrm{m} / \mathrm{N}]$ & 3.82 & $\pm 0.27(7.0 \%)$ \\
\hline $\mathrm{k}_{6},[\mathrm{rad} \times \mu \mathrm{m} / \mathrm{N}]$ & 4.01 & $\pm 0.35(8.7 \%)$ \\
\hline
\end{tabular}

\section{CONCLUSIONS}

The paper presents a new approach for the modelling and identification of the elastostatic parameters of heavy industrial robots with the pneumatic gravity compensator. It proposes a methodology and data processing algorithms for the identification of elastostatic parameters of gravity compensator and manipulator. To increase the identification accuracy, the design of experiments has been used aimed at proper selection of the measurement configurations. In contrast to other works, it is based on the industry oriented performance measure that is related to the robot accuracy under the loading. The advantages of the developed techniques are illustrated by experimental study of the industrial robot Kuka KR-120, for which the joint compliances and parameters of the gravity compensator have been identified.

\section{ACKNOWLEDGMENTS}

The work presented in this paper was partially funded by Innopolis University and the project Partenariat Hubert $\mathrm{Cu}$ rien SIAM 2016 France-Thailand.

\section{REFERENCES}

[1] W. Zhu, W. Qu, L. Cao, D. Yang, and Y. Ke, "An off-line programming system for robotic drilling in aerospace manufacturing," The International Journal of Advanced Manufacturing Technology, vol. 68 , pp. 2535-2545, 2013.

[2] M. Guillo and L. Dubourg, "Impact \& improvement of tool deviation in friction stir welding: Weld quality \& real-time compensation on an industrial robot," Robotics and Computer-Integrated Manufacturing, vol. 39, pp. 22-31, 6// 2016.

[3] B. Denkena and T. Lepper, "Enabling an Industrial Robot for Metal Cutting Operations," Procedia CIRP, vol. 35, pp. 79-84, // 2015.

[4] C. Dumas, S. Caro, M. Cherif, S. Garnier, and B. Furet, "Joint stiffness identification of industrial serial robots," Robotica, vol. 30, pp. 649-659, 2012.

[5] A. Nubiola and I. A. Bonev, "Absolute calibration of an ABB IRB 1600 robot using a laser tracker," Robotics and Computer-Integrated Manufacturing, vol. 29, pp. 236-245, 2013.

[6] G. Alici and B. Shirinzadeh, "Enhanced stiffness modeling, identification and characterization for robot manipulators," Robotics, IEEE Transactions on, vol. 21, pp. 554-564, 2005.

[7] A. Klimchik, D. Chablat, and A. Pashkevich, "Stiffness modeling for perfect and non-perfect parallel manipulators under internal and external loadings," Mechanism and Machine Theory, vol. 79, pp. 1$28,9 / / 2014$

[8] U. Schneider, M. Momeni-K, M. Ansaloni, and A. Verl, "Stiffness modeling of industrial robots for deformation compensation in machining," in 2014 IEEE/RSJ International Conference on Intelligent Robots and Systems, 2014, pp. 4464-4469.

[9] A. Klimchik, D. Bondarenko, A. Pashkevich, S. Briot, and B. Furet, "Compliance Error Compensation in Robotic-Based Milling," in Informatics in Control, Automation and Robotics. vol. 283, J.-L. Ferrier, A. Bernard, O. Gusikhin, and K. Madani, Eds., ed: Springer International Publishing, 2014, pp. 197-216.

[10] A. Klimchik, A. Pashkevich, D. Chablat, and G. Hovland, "Compliance error compensation technique for parallel robots composed of non-perfect serial chains," Robotics and ComputerIntegrated Manufacturing, vol. 29, pp. 385-393, 4// 2013.

[11] N. R. Slavkovic, D. S. Milutinovic, and M. M. Glavonjic, "A method for off-line compensation of cutting force-induced errors in robotic machining by tool path modification," The International Journal of Advanced Manufacturing Technology, vol. 70, pp. 2083-2096, 2013.

[12] A. Klimchik, B. Furet, S. Caro, and A. Pashkevich, "Identification of the manipulator stiffness model parameters in industrial environment," Mechanism and Machine Theory, vol. 90, pp. 1-22, 8// 2015. 
[13] Y. Wu, A. Klimchik, S. Caro, B. Furet, and A. Pashkevich, "Geometric calibration of industrial robots using enhanced partial pose measurements and design of experiments," Robotics and Computer-Integrated Manufacturing, vol. 35, pp. 151-168, 10// 2015.

[14] J. Hollerbach, W. Khalil, and M. Gautier, "Model Identification," in Springer Handbook of Robotics, B. Siciliano and O. Khatib, Eds., ed: Springer Berlin Heidelberg, 2008, pp. 321-344.

[15] A. Klimchik, Y. Wu, C. Dumas, S. Caro, B. Furet, and A. Pashkevich, "Identification of geometrical and elastostatic parameters of heavy industrial robots," in IEEE International Conference on Robotics and Automation (ICRA) 2013, pp. 3707-3714.

[16] A. Klimchik, S. Caro, Y. Wu, D. Chablat, B. Furet, and A. Pashkevich, "Stiffness Modeling of Robotic Manipulator with Gravity Compensator," in Computational Kinematics. vol. 15, F. Thomas and A. Perez Gracia, Eds., ed: Springer Netherlands, 2014, pp. 185-192.

[17] V. Arakelian, "Gravity compensation in robotics," Advanced Robotics, vol. 30, pp. 79-96, 2016.

[18] C. Cho and S. Kang, "Design of a Static Balancing Mechanism for a Serial Manipulator With an Unconstrained Joint Space Using OneDOF Gravity Compensators," IEEE Transactions on Robotics, vol. 30, pp. 421-431, 2014.

[19] A. De Luca and F. Flacco, "A PD-type regulator with exact gravity cancellation for robots with flexible joints," in Robotics and Automation (ICRA), 2011 IEEE International Conference on, 2011, pp. 317-323.

[20] A. De Luca, B. Siciliano, and L. Zollo, "PD control with on-line gravity compensation for robots with elastic joints: Theory and experiments," Automatica, vol. 41, pp. 1809-1819, 10// 2005.

[21] S.-F. Chen and I. Kao, "Conservative congruence transformation for joint and Cartesian stiffness matrices of robotic hands and fingers," The International Journal of Robotics Research, vol. 19, pp. 835-847, 2000.

[22] J. K. Salisbury, "Active stiffness control of a manipulator in Cartesian coordinates," in Decision and Control including the Symposium on Adaptive Processes, 1980 19th IEEE Conference on, 1980, pp. 95100.

[23] S. J. Yan, S. K. Ong, and A. Y. C. Nee, "Stiffness analysis of parallelogram-type parallel manipulators using a strain energy method," Robotics and Computer-Integrated Manufacturing, vol. 37, pp. 13-22, 2// 2016.

[24] Y. Li and Q. Xu, "Stiffness analysis for a 3-PUU parallel kinematic machine," Mechanism and Machine Theory, vol. 43, pp. 186-200, 2008.

[25] D. Deblaise, X. Hernot, and P. Maurine, "A systematic analytical method for PKM stiffness matrix calculation," in Robotics and Automation, 2006. ICRA 2006. Proceedings 2006 IEEE International Conference on, 2006, pp. 4213-4219.

[26] C. Gosselin and D. Zhang, "Stiffness analysis of parallel mechanisms using a lumped model," International Journal of Robotics and Automation, vol. 17, pp. 17-27, 2002.

[27] J.-P. Merlet and C. Gosselin, "Parallel Mechanisms and Robots," in Springer Handbook of Robotics, B. Siciliano and O. Khatib, Eds., ed: Springer Berlin Heidelberg, 2008, pp. 269-285.

[28] A. Klimchik and A. Pashkevich, "Serial vs. quasi-serial manipulators: Comparison analysis of elasto-static behaviors," Mechanism and Machine Theory, vol. 107, pp. 46-70, 1// 2017.

[29] K. Subrin, L. Sabourin, R. Cousturier, G. Gogu, and Y. Mezouar, "New Redundant Architectures in Machining: Serial and Parallel Robots," Procedia Engineering, vol. 63, pp. 158-166, // 2013.

[30] Y. Guo, S. Yin, Y. Ren, J. Zhu, S. Yang, and S. Ye, "A multilevel calibration technique for an industrial robot with parallelogram mechanism," Precision Engineering, vol. 40, pp. 261-272, 4// 2015.

[31] B. Vemula, G. Spampinato, T. Brogardh, and X. Feng, "Stiffness Based Global Indices for Structural Evaluation of Anthropomorphic Manipulators," in ISR/Robotik 2014; 41st International Symposium on Robotics; Proceedings of, 2014, pp. 1-8.

[32] A. Pashkevich, A. Klimchik, and D. Chablat, "Enhanced stiffness modeling of manipulators with passive joints," Mechanism and machine theory, vol. 46, pp. 662-679, 2011.

[33] A. Pashkevich, D. Chablat, and P. Wenger, "Stiffness analysis of overconstrained parallel manipulators," Mechanism and Machine Theory, vol. 44, pp. 966-982, 2009.
[34] A. Klimchik, A. Pashkevich, S. Caro, and D. Chablat, "Stiffness matrix of manipulators with passive joints: computational aspects," Robotics, IEEE Transactions on, vol. 28, pp. 955-958, 2012. 